\title{
Evaluación de satisfacción de los estudiantes sobre las clases virtuales
}

\author{
Bautista Isaac ${ }^{1}$, Carrera Giulianna ${ }^{2}$, León Emily ${ }^{3}$ y Laverde David ${ }^{4}$. \\ \{isaac.bautista ${ }^{1}$, giulianna.carrera ${ }^{2}$, emily.leon ${ }^{3}$, david.laverde ${ }^{4}$ @ 0 udla.edu.ec. \\ https://orcid.org/0000-0002-6970-9523 $3^{1}$, https://orcid.org/0000-0002-6280-79992, \\ https://orcid.org/0000-0003-1395-37353, https://orcid.org/0000-0003-3841-86164 \\ Universidad de las Américas \\ Quito -Ecuador
}

Recibido (01/07/20), Aceptado (17/07/20)

\begin{abstract}
Resumen: Las clases virtuales son una modalidad de estudio a distancia que ha sido aplicadas por más de 10 años. Son utilizadas principalmente en universidades para abarcar las necesidades de sus estudiantes que no pueden acceder al sistema presencial. Al encontrarnos en una emergencia sanitaria por el COVID-19, la aplicación de las clases virtuales alrededor del mundo se volvió una obligación para precautelar la vida de los estudiantes. Es por esto que la población universitaria tuvo que adaptarse a nuevas condiciones de estudio como: las clases, trabajos y exámenes en línea con el fin de no detener su aprendizaje. Sin duda, esto desencadenó inconformidades e inquietudes al estudiar por primera vez bajo esta modalidad. En este trabajo se evaluó la satisfacción sobre clases virtuales tomadas el primer semestre del 2020 a estudiantes de la carrera de Ingeniería Industrial de la Universidad de las Américas con edades entre 18 a 20 años. Para ello se realizó una encuesta de 15 ítems los cuales evaluaron las distintas condiciones que se presentaron en las clases virtuales durante el periodo de pandemia, con datos validados por un alfa de Cronbach 0,92 . En este trabajo se obtuvo como resultado que gran parte de las personas encuestadas aprobó el software aplicado para la realización de las clases virtuales debido a que es una herramienta de uso intuitivo y de fácil acceso, por otro lado, están en desacuerdo con la modalidad de evaluación del conocimiento adquirido.
\end{abstract}

Palabras Clave: Clases virtuales; Satisfacción estudiantil; COVID-19; TIC's.

\section{Evaluation of student satisfaction with virtual classes}

\begin{abstract}
Virtual classes are a form of distance study that has been applied for more than 10 years. It is mainly used in universities to cover the needs of its students who cannot access the face-to-face system. As we are in a health emergency due to COVID-19, the application of virtual classes around the world became an obligation to protect the lives of students. This is why the university population had to adapt to new study conditions such as online classes, jobs and exams in order not to stop their learning. Without a doubt, this triggered disagreements and concerns when studying for the first time under this modality. In this work, satisfaction about virtual classes taken in the first semester of 2020 was evaluated for students of the Industrial Engineering career at the Universidad de las Américas, with ages between 18 and 20 years old. For this, a 15-item survey was carried out, which evaluated the different conditions that occurred in virtual classes during the pandemic period, with data validated by a Cronbach's alpha of 0.92. In this work it was obtained as a result that a large part of the surveyed people approved the software applied for the realization of the virtual classes because it is an intuitive and easily accessible tool, on the other hand, they disagree with the modality of evaluation of acquired knowledge.
\end{abstract}

Keywords: Virtual classes; Student satisfaction; COVID-19; TIC.

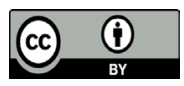




\section{I.INTRODUCCIÓN}

La tecnología ha tenido un avance significativo en todas sus ramas, las cuales se han ido perfeccionando con el fin de obtener resultados mucho más eficientes. La educación forma parte de esta evolución en crecimiento tecnológico, implementando nuevas maneras de enseñar y nuevas formas de aprender alrededor del mundo [1] Las clases virtuales son la educación del futuro, teniendo un alcance a grandes distancias y en los distintos lugares a través del mundo con solo poseer una red inalámbrica de internet. En un mundo globalizado y conectado a través de internet, la comunicación se ha vuelto mucho más veloz, las noticias llegan de manera inmediata y por tanto también la educación logra llegar a los que la necesitan. Así es como se llegan a crear los entornos virtuales de aprendizaje (EVA), los cuales, según Belloch, [2] es un software denominado plataforma virtual el cual permite la formación en línea. Mediante esta plataforma el estudiante puede recopilar información, obtener recursos de aprendizaje y evaluar su conocimiento adquirido. Este sistema es comúnmente implementado por universidades alrededor del mundo que prestan sus servicios de educación a distancia o educación semipresencial.

En el presente año 2020 en que se realiza este trabajo el mundo vive una pandemia que ha atacado y detenido las actividades cotidianas del ser humano, siendo una de ellas la educación primaria, secundaria y superior; recurriendo así a la implantación de clases virtuales. Según el informe nacional del Ecuador, existen 59 universidades alrededor del país y más de 500 mil estudiantes cursando una carrera universitaria [3], estos tuvieron que adaptarse al nuevo sistema de educación en línea con el fin de no detener las clases y el aprendizaje por la pandemia. La Universidad de las Américas es una de las tantas que se acogió al sistema educativo en línea para sus aproximadamente 17 mil estudiantes.

Sin embargo, este sistema causó sorpresa e incertidumbre para muchos estudiantes ya que no todos han recibido clases con esta modalidad; por lo que a lo largo del semestre han surgido diversas inconformidades e inquietudes en base a la modalidad de evaluación de aprendizaje, el sistema de educación en línea y los resultados de aprendizaje (RDA). Este trabajo estuvo centrado en conocer la satisfacción de los estudiantes sobre las clases virtuales en el primer semestre del 2020. En base al problema, se tiene como objetivo general conocer la satisfacción de los estudiantes sobre las clases virtuales dictadas en el primer del semestre 2020.

Para obtener los resultados pertinentes, el trabajo está fundamentado en una encuesta de satisfacción que consta de 15 preguntas relaciones al tema con 5 opciones de respuesta, las cuales una vez analizadas fueron validadas por el estudio del Alfa de Cronbach obteniendo un valor de 0,92 lo cual garantiza la confiabilidad de la información y permite realizar un análisis y propuestas de mejora acerca de las clases virtuales. La encuesta fue aplicada a un total de 58 estudiantes pertenecientes a la carrera de Ingeniería Industrial de la Universidad de las Américas.

Es trabajo está compuesto por cinco secciones, en la primera se expone la introducción, como segunda parte se describe el desarrollo teórico acerca de las clases virtuales. En la tercera sección se plantea la metodología utilizada para el desarrollo de los resultados, mientras que en la cuarta ya se exponen los resultados obtenidos en el análisis del trabajo; por último, en la quinta sección se muestran las conclusiones planteadas en base al análisis de los resultados.

\section{II.DESARROLLO}

\section{A.El comienzo de las clases virtuales}

La educación virtual es empleada por primera vez a mediados de los años 90 y desde ese momento se ha convertido en un método común de aprendizaje a distancia. Las primeras referencias de educación virtual en literatura datan de 1995 en dos provincias de Canadá, específicamente en Alberta y Newfoundland/Labrador. Las primeras escuelas virtuales de Alberta se centran en zonas rurales, las mismas que en 4 años fueron expandiendo al alrededor de 23 programas. A partir de 1996 estas no eran las únicas provincias que querían implementar el teleaprendizaje, se unió Terranova y se creó el Centrefor Distance Learning and Innovation que permitió que las inscripciones de este método de estudio incrementasen de 200 a 1500. En 2003 por medio de una encuesta nacional se comprobaba que Alberta era la provincia que contaba con más estudiantes aprendiendo virtualmente, años después se comienzan a crear nuevos programas de apoyo en otras provincias de Canadá como Learnnow BC con el propósito de que los estudiantes tengan mayor flexibilidad y más opciones de cursos. En 1997 se crean las primeras dos escuelas virtuales en los Estados Unidos expandiéndose posteriormente a no menos de 30 estados; su continua expansión permitió llegar a 11 países extranjeros y a permitir un aumento radical en el número de 
inscripciones de alumnos en cursos en línea, es más, el gobierno de Michigan hizo que sea obligatorio tomar al menos un curso en línea para que sus estudiantes se gradúen [4].

\section{B.Herramientas online para el uso en clases virtuales}

La videoconferencia con un tutor llega a ser un pilar importante en el aprendizaje en la modalidad no presencial, el uso de esta implica un cambio de paradigma con respecto a esta herramienta por parte de quienes la utilizan exigiendo una transformación del acto educativo. Se establece a la videoconferencia como el recurso que permite mantener el contacto entre profesor y alumnos, desde un punto de vista de la docencia, es un cambio radical y por lo tanto conlleva un proceso de adaptación y cambio de metodología según las características y posibilidades que ofrece la aplicación a usar, sin embargo, implementar el uso eficaz de tecnología en el ámbito educativo exige una revolución en la forma de entender la enseñanza y aprendizaje [5].

Una de las herramientas más utilizadas por las organizaciones educativas es "Moodle", con la cual los docentes buscan elaborar sistemas que promuevan una evaluación formativa en lugar de pruebas automatizadas, enfocándose principalmente en la retroalimentación, así como el análisis y reflexión de la práctica educativa. Estas y varias herramientas como Office 365, Zoho, Google Apps for Education, Edmodo, entre otras ayudan con la planificación y diseño de experiencias presentadas a los estudiantes dando priorización a la evaluación del aprendizaje [6].

Adicionalmente, en los últimos años, los cursos masivos abierto en línea (MOOC, por sus siglas en inglés), llegan a ser una herramienta de enseñanza muy útil, ya que no existen limitaciones de horarios, idiomas o ubicación geográfica, solo requieren de un dispositivo electrónico que tenga acceso a internet, por ello se ha descrito a los MOOCs como una respuesta eficiente a la actual emergencia sanitaria. Por ello plataformas como Coursera, edX, FutureLearn, XuentangX, Udacity, Miríadax, Alison, Fun.MOOC, UPV [X], Canvas Network, OpenWHO, Formación Alcalá, Open2Study, Class Central y MOOC List, ofrecen sus cursos online y posible diplomado con un costo adicional, en suma, actualmente se ha agregado contenido sobre la COVID-19, en donde a la fecha de 15 de abril del 2020, se han encontrado 28 MOOCs de inscripción gratis [7].

La única restricción de estos cursos gratuitos y del aprendizaje en general con ayuda de herramientas online son el idioma, disponibilidad de acceso a internet y el grado de alfabetización digital de la población, como en Ecuador nos encontramos en una creciente conciliación de tecnología frente a la pandemia, las herramientas online de aprendizaje en universidades, escuelas y colegios, así también como los MOOC, son alternativas viables para la formación de profesionales durante el aislamiento social.

\section{C.Herramientas online en la mejora de las actividades académicas}

Sin lugar a dudas, el internet está revolucionando al mundo de manera vertiginosa a través de continuos avances sustanciales que han traspasado límites inimaginables hasta hace algunos años atrás. El progreso masivo de las Tecnologías de la Información y Comunicación (TIC's) continúa cambiando la manera tradicional en la que los diferentes conocimientos eran elaborados, adquiridos y transmitidos a otras personas. Es por ello que resulta imperativo que los sistemas y modelos educativos se adapten a estas modificaciones dentro de una sociedad que día tras día se encuentra más sumergida en las TIC’s [8], con el fin de establecer e implementar estrategias oportunas que permitan hacer un uso adecuado y efectivo de estas herramientas e instrumentos para aprovechar las facilidades que ofrece y potenciar la enseñanza, el aprendizaje y el conocimiento.

El uso de las herramientas online en el ámbito educativo trae consigo numerosos beneficios ya que brinda la posibilidad de innovar el contenido de los diferentes cursos, así como los métodos pedagógicos empleados en los mismos. Para alcanzar un mejor nivel educativo dentro de un mundo altamente competitivo y exigente es necesario el apoyo de recursos y materiales didácticos que faciliten el proceso de enseñanza de los alumnos. Estas herramientas permiten incentivar y guiar al estudiante en la construcción del conocimiento y el pensamiento crítico; motivando en ellos aspectos importantes en su formación personal y académica como la creatividad, el ingenio y el autoaprendizaje. Por otra parte, la virtualización de las actividades académicas cambia el entorno físico de las clases presenciales por uno virtual en la formación en línea. Esto requiere de canales de comunicación efectivos que generen un vínculo sólido entre los profesores y alumnos que trascienda el espacio material del aula y los motive a empoderarse del conocimiento [9].

Las herramientas virtuales poseen una poderosa capacidad de reducir la brecha entre los estudiantes para socializar la información de manera ágil y sencilla. Son de gran utilidad en el incremento del rendimiento académi- 
co de los estudiantes en diversos niveles educativos; mejorando el estado de conocimiento, así como la compresión y aplicación de conocimientos en múltiples disciplinas del saber [9]. Más allá de eso, el uso de estas tecnologías fomenta la autonomía y la participación activa, mejora la comunicación, genera una formación flexible, dinámica e interactiva y brinda un acceso fácil y rápido a cualquier contenido de interés para quien lo necesite en cuestión de solo unos cuantos clicks. Como es evidente, las Tecnologías de la Información y Comunicación han venido para quedarse y es de suma importancia aprovechar al máximo los beneficios y facilidades que ofrecen para garantizar una educación de calidad que permita mejorar el aprendizaje, la enseñanza y los procesos formativos en los distintos niveles de educación.

\section{D.Clases virtuales por la pandemia}

La pandemia del COVID-19 (Coronavirus) logró expandirse alrededor del mundo y Ecuador no fue la excepción en el alto índice de contagios y muertes. Es por esto que el gobierno aplicó medidas similares ya utilizadas alrededor del mundo con el fin de detener el contagio; una de ellas fue la suspensión de la jornada académica presencial dando paso a la educación por medio de clases virtuales. En el tiempo actual en que vivimos los estudiantes manejan recursos de investigación búsqueda bibliográfica por medio de redes de internet globales que les permiten acceder de manera rápida a los resultados que buscan [10].

Las clases virtuales pasaron de ser una opción de tipo de estudio a una modalidad obligatoria con el fin de no detener el aprendizaje estudiantil debido a la pandemia. Más del 80\% de la población universitaria del Ecuador realiza sus estudios de educación superior de manera presencial, por lo que a la mayoría le toco adaptarse a este nuevo sistema temporal, aprendiendo a manejar las distintas plataformas virtuales, rindiendo evaluaciones con software por medio de internet y auto educándose mediante el uso de redes inalámbricas y bibliotecas virtuales. Al vivir esta primera experiencia virtual existieron inquietudes o inconformidades con el sistema ya que no es una forma de estudio común o de uso frecuente. El tiempo es uno de los factores críticos de esta modalidad, ya que al depender del internet puede que la señal se debilite o desaparezca [5]. Esto causa problemas por ejemplo al estar rindiendo una evaluación o examen debido a que puede suspenderse o afectar el desarrollo del mismo. Por el contrario, en una clase presencial esto no sucedería ya que el examen se lo realiza en hoja.

Sin embargo, el sistema virtual también ha tenido sus beneficios dentro del impacto de estudio debido a las circunstancias que se viven. El estudiante puede repetir las clases del día ya que son grabadas en su totalidad, lo que le permite tener un mayor refuerzo de la materia. De igual forma la comunicación entre estudiante y docente es más directa y rápida debido al internet, permitiendo estar en contacto si existe alguna duda o problema [5]. De esta forma las clases virtuales tratan de brindar el mayor apoyo posible al estudiante con el objetivo que se asemejen lo al sistema presencial.

\section{III.METODOLOGÍA}

La metodología empleada para este trabajo fue una encuesta, la cual nos permitió obtener información objetiva sobre la opinión de los estudiantes acerca del aprendizaje virtual y las clases impartidas bajo esta modalidad durante el primer semestre del año 2020. La recopilación de datos se obtuvo a partir de un cuestionario realizado a una muestra de 58 estudiantes de la Universidad de las Américas pertenecientes a la carrera de Ingeniería Industrial, cuyo rango de edad oscila entre 18 y 20 años.

El cuestionario está conformado por 15 ítems, los cuales debían ser calificadas seleccionando una de las 5 opciones de respuesta, siendo 5 el mayor nivel y 1 el menor nivel de satisfacción. Entre los temas abordados se encuentra: el uso de Microsoft Teams y Smowl, la facilidad de comprensión, el aprendizaje, los recursos y materiales, cantidad de trabajo autónomo, flexibilidad horaria, el tiempo y dedicación por parte de los profesores, la motivación, la sinergia entre estudiantes, el sistema evaluativo y por último la efectividad de la modalidad online. La encuesta fue desarrollada en la aplicación Google Forms y el tiempo para completarla no superaba los 2 minutos ya que eran preguntas de fácil comprensión acerca del tema de interés actual. Los estudiantes tuvieron un lapso de tiempo de 5 días para responder la encuesta, cual nos permitió recolectar la información necesaria para los cálculos posteriores.

Una vez obtenidos los datos pertinentes fue posible realizar el estudio del alfa de Cronbach, el cual nos permite identificar si el instrumento aplicado para la evaluación de los resultados compila información defectuosa que nos dirige a conclusiones equivocadas o, por el contrario, si se trata de un instrumento fiable que hace mediciones sólidas y consistentes. La fórmula empleada se define de la siguiente manera: 


$$
\alpha=\frac{k}{k-1} *\left(1-\frac{\sum V i}{V t}\right)
$$

Donde:

$\mathrm{k}=$ Número de ítems

$\mathrm{Vi}=$ Varianza de ítems

$\mathrm{Vt}=$ Varianza del puntaje total

$\alpha=$ Alfa de Cronbach

En el presente estudio al realizar el respectivo cálculo del alfa de Cronbach se obtuvo un valor de 0,92 que sustenta la confiabilidad de los datos recopilados.

\section{IV.RESULTADOS}

A continuación, se presenta un diagrama de barras de las respuestas promedio de los 15 ítems.

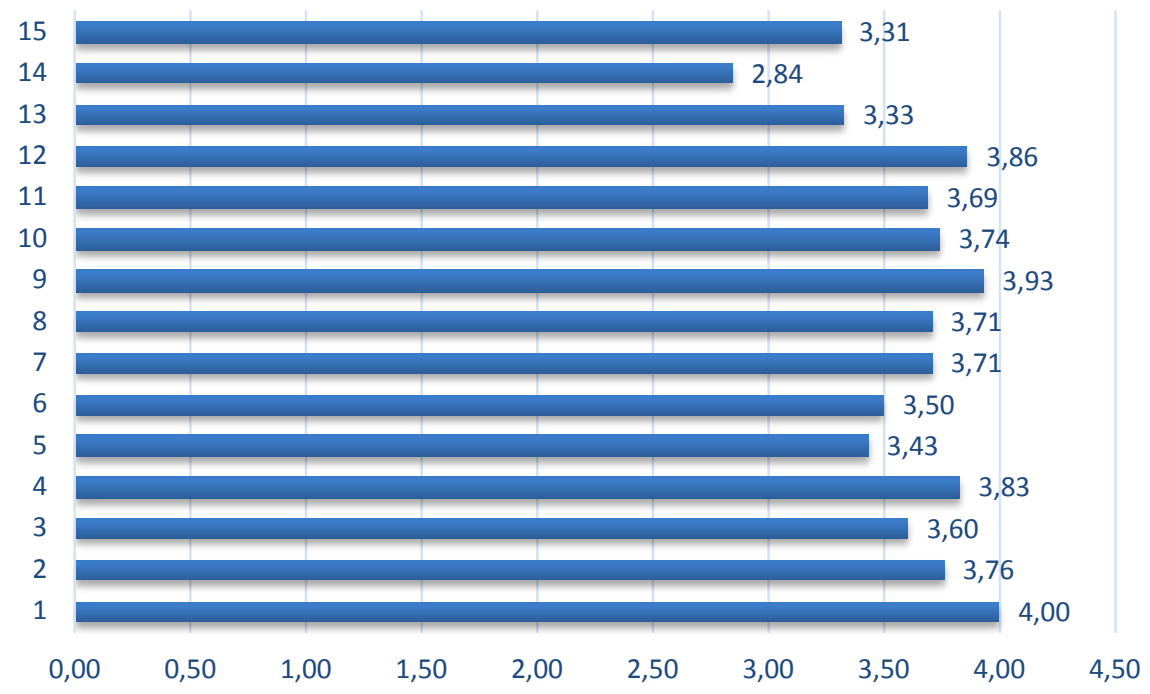

Fig. 1. Diagrama de respuestas promedio

Una vez realizado el análisis de las respuestas obtenidas en el instrumento de investigación se pudo constatar que el ítem 1 correspondiente al uso del software Microsoft Teams como herramienta adecuada para el aprendizaje virtual, tuvo una gran aceptación por parte de los encuestados. Por otro lado, el ítem que obtuvo mayor rechazo al obtener una amplia cantidad de respuestas insatisfactorias fue el ítem número 14 referente a el uso de la herramienta Smowl como instrumento de monitoreo durante los exámenes virtuales. Adicionalmente se puede destacar que todas las respuestas promedio se encuentran por encima de la media de satisfacción, por lo que se puede concluir que las clases impartidas en el primer semestre del año 2020 tuvieron una aceptable acogida por parte de los encuestados.

En los siguientes gráficos se puede observar el porcentaje con base a las 5 opciones de respuesta de acuerdo a los niveles de satisfacción establecidos por cada encuestado. Considerando los ítems 1 y 14 como aquellos con el mayor y menor índice de satisfacción respectivamente. 


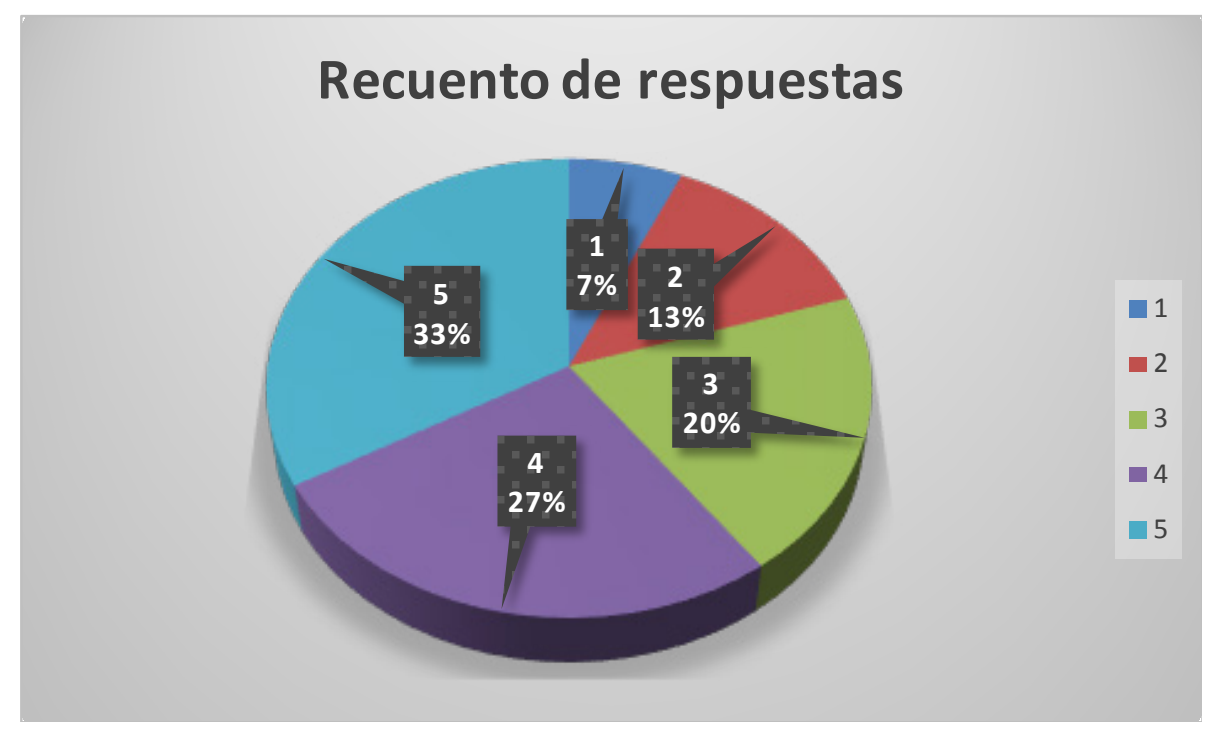

Fig. 2. Gráfica Recuento Ítem 1

Los datos representados gráficamente indican que únicamente 2 de las 58 personas encuestadas afirman que la herramienta Microsoft Teams es inadecuada para el aprendizaje virtual. Mientras que 27 de ellas aprueban su uso para estos propósitos.

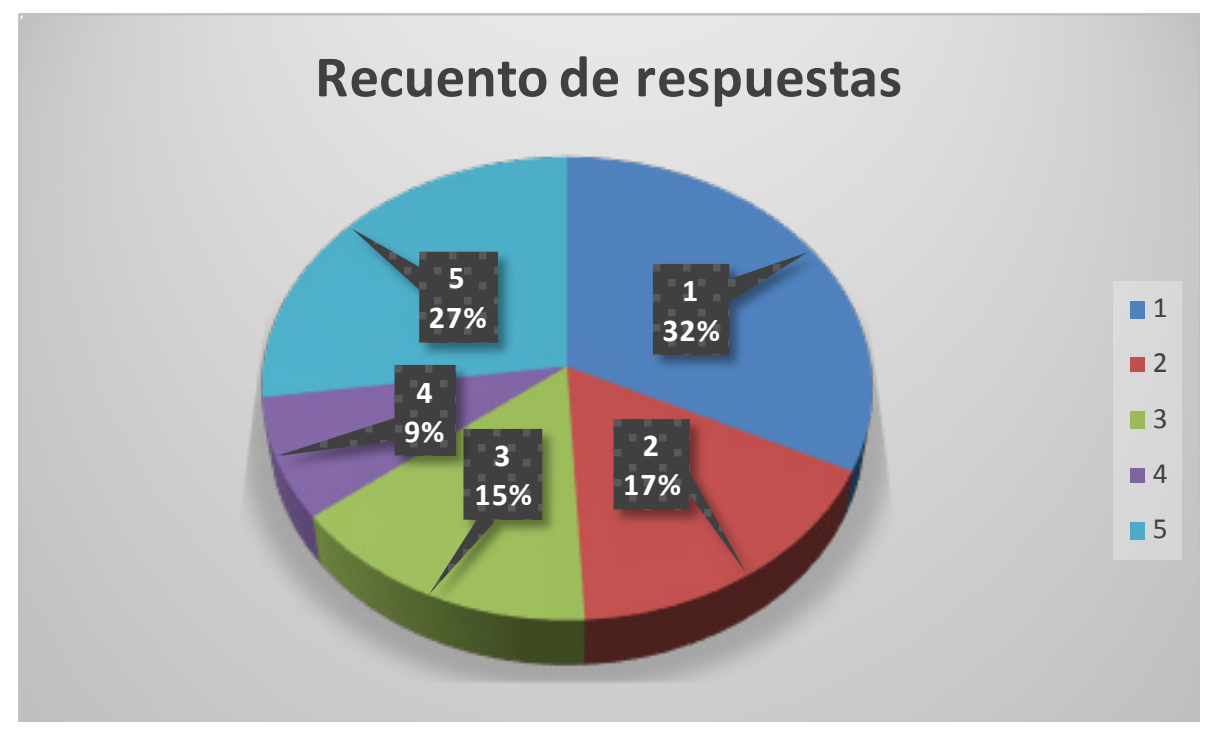

Fig. 3. Gráfica Recuentro Ítem 14

En la gráfica circular se puede corroborar que 19 personas de la muestra total consideran el uso del sistema Smowl como una herramienta innecesaria durante las evaluaciones online. Por el contrario, 16 personas opinan que resulta imprescindible el monitoreo continuo del estudiante mientras desarrolla sus exámenes.

\section{V.CONCLUSIONES}

Una vez realizado el análisis de los resultados obtenidos en base a la encuesta aplicada se concluye que el aprendizaje bajo la modalidad virtual se percibe de distintas maneras por parte de los estudiantes, quienes han tenido que adaptarse a los cambios y nuevas metodologías para continuar desarrollando sus conocimientos y habilidades a pesar de las dificultades y complicaciones asociadas a la crisis sanitaria por la pandemia. La opinión de los encuestados permitió conocer el nivel de satisfacción acerca de algunos componentes que engloban la educación en línea con el fin de constatar la efectividad de las clases online, el aprendizaje adquirido y el sistema evaluativo. La importancia del estudio radica en que, conociendo estos criterios, es posible efectuar acciones de mejora que impulsen 
el desarrollo eficiente y eficaz de estas herramientas tecnológicas para garantizar continuamente la calidad de la educación si la emergencia sanitaria se mantiene. Esto permitirá una mejor adaptación por parte de los estudiantes, estimulando su deseo de aprender y posiblemente incrementando los niveles de satisfacción a futuro en torno a la nueva modalidad de aprendizaje.

En conclusión, el análisis realizado en este trabajo demuestra la aceptación por parte de las personas encuestadas acerca del uso de la plataforma Microsoft Teams para el desarrollo y planteamiento de las clases virtuales realizadas en el primer semestre del año 2020. Al tener estos resultados se aconseja que en próximas circunstancias o incluso para el uso de clases a distancia se utilice esta herramienta ya que no existe un alto porcentaje de inconformidad con el uso de la misma en el desarrollo de las clases indistintamente de la materia dictada. Por lo que sin duda este trabajo permite identificar la satisfacción obtenida por los estudiantes con base en las herramientas proporcionadas para cursar un semestre en medio de una emergencia sanitaria.

Mientras que el uso de Smowl se encuentra más dividido, ya que no es mucha la diferencia entre el número de encuestados con mayor y menor nivel de satisfacción con esta herramienta. Sin embargo, se puede concluir que los estudiantes no están satisfechos con la utilización de este sistema de reconocimiento facial durante las evaluaciones de algunas materias. Debido a que la diferencia es de apenas un $5 \%$ se considera que las autoridades deberían tomar la decisión de seguir o no con su implementación, cambiarlo por otro recurso o confiar en los valores de tenga cada uno de los estudiantes.

\section{REFERENCIAS}

[1]K. Brandl, «Are you ready to Moodle?,» Language Learning \& Technology, vol. 9, no 2, pp. 16-23, 2005.

[2]C. Belloch, «Entornos virtuales de aprendizaje,» Universidad de Valencia, vol. 1, nº 1, pp. 1-3, 2012.

[3]J. Jarrín, «Informe Nacional: Ecuador,» Universa, Quito, 2016.

[4]M. Reeves. and. T. Barbour, «The reality of virtual schools: A review of the literature,» Computers \& Education, vol. 52, no 10, pp. 402-416, 2009.

[5]C. Bonfill, «Clases virtuales a través de videoconferencias: factores críticos vivenciados por los tutores en un sistema de educación a distancia.,» Temas de Managment, vol. 5, n 2, pp. 12-20, 2007.

[6]A. García, M. Mediavilla and A. Casares, «Evaluación entre iguales en entornos de edcucación superior online mediante el taller Moodle,» A case study, vol. 13, n 2, pp. 119-126, 2020.

[7]R. Bendezu, A. Quijuano, H. Rebatta and G. Gutierrez, «Aprendizaje en tiempos de aislamiento social: cursos masivos abiertos en línea sobre la COVID-19,» vol. 37, n 2, pp. 375-277, 2020.

[8]M. Bautista and R. Torres, «El uso de material didáctico y las tecnologías de la información y comunicación(TIC's) para mejorar el alcance académico,» Ciencia y tecnología, nº 14, 2014.

[9]G. Gutierrez, P. Krystell, C. Zarate, I. Juarez. and M. López, «Uso de tecnologías de la información en el rendimiento académico basado en una población de estudiantes de Medicina,» Educ Med Super, vol. 31, n 2, pp. 23-34, 2017.

[10]A. Rodrífuez, B. González y J. Fariñas, «Simulaciones virtuales como complemento de las clases y los laboratorios de Física. Ejemplos en la carrera de Ingeniería en Telecomunicaciones y Electrónica.,» Latin-American Journal of Physics Education, vol. 7, nº 4, pp. 16-20, 2013.

\section{RESUMEN CURRICULAR}

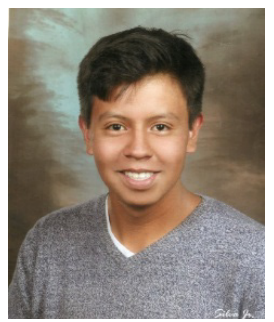

David Laverde, de nacionalidad ecuatoriana, Bachiller Técnico en Servicios Contables de la Unidad Educativa Borja 3 Cavanis, actualmente estudiante de la carrera de Ingeniería Industrial en la Universidad de las Américas 


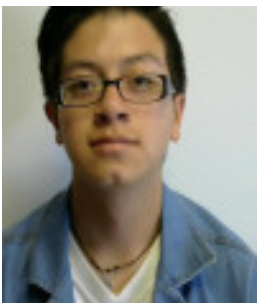

Is aac Bautista, de nacionalidad ecuatoriana, Bachiller en ciencias, graduado de la Unidad Educativa Particular Isaac Newton, actualmente estudiante de la carrera de Ingeniería Industrial en la Universidad de las Américas

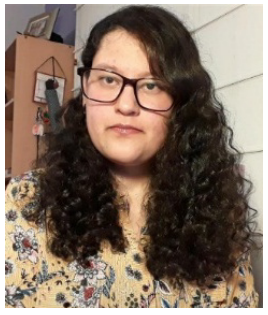

Giulianna Carrera, de nacionalidad ecuatoriana, Bachiller Internacional Físico-Químico, graduada de la Unidad Educativa Particular Isaac Newton, actualmente estudiante de la carrera de Ingeniería Industrial en la Universidad de las Américas.

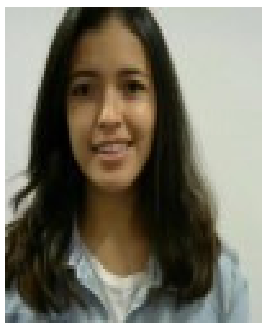

Emily León, de nacionalidad ecuatoriana, Bachiller en Ciencias Generales graduada de la Unidad Educativa Cardenal Spellman Femenino, actualmente estudiante de la carrera de Ingeniería Industrial en la Universidad de las Américas 